\title{
Leukocyte morphology on an anti-CD antibody microarray for acute leukemia diagnosis: morphology rejuvenated
}

Keywords: leukocyte morphology, antibody microarray, cytochemistry, flow cytometry, acute leukemia, antibody microarray

Abbreviations: CD, cluster of differentiation; ALL, acute lymphoblastic leukemia; AML, acute myeloid leukemia; WHO, world health organization

\section{Opinion}

"Morphology is an art, a bit like dancing. It is difficult to learn. There are people who never learn to dance." Prof. Torsten Haferlach, from a talk at EHA-SWG Scientific Meeting on Integrated Diagnosis Strategies in Oncohematology for the Management of Cytopenias and Leukocytosis.

Acute leukemia diagnosis steadily shifts towards automated analysis and precise molecular methods banishing the methods resistant to complete automatization (such as cytochemistry and bone marrow morphology). The reasons for this lie above all in the desire to eliminate the human factor as well as to find a way to cope with increasing work load. The better understanding of the biology of the disease achieved with the advanced molecular technologies led to the inclusion of new recurrent cytogenetic abnormalities, mutations and gene fusions into the revised WHO classification of tumors of the hematopoietic and lymphoid tissues. Wherever possible the acute leukemias are classified by mutations rather than by underlying subtypes according to French-American-British (FAB) classification. ${ }^{1}$ $\mathrm{FAB}$ classification is retained only in the "not otherwise specified" category. These changes give a probably involuntary message that as mutations prevail over morphologic and immunophenotypic characteristics of leukemic cells the latter at a certain point will not be needed for the diagnosis.

On the other hand, as the amount of diagnostic methods involved in acute leukemia diagnosis increases, there is a rise in demand for cost-optimizing strategies, and FAB classification ${ }^{2}$ is used to narrow the range of molecular tests to be performed on any particular bone marrow sample.

According to the established guidelines ${ }^{3,4}$ the diagnosis of acute leukemia starts with the analysis of morphology, cytochemistry and immunophenotype of the bone marrow aspirate. If more than $20 \%$ of the total nucleated cells in bone marrow aspirate are immature leukocyte precursors (blasts), the patient is diagnosed with acute leukemia. Leukemia subtype is than determined by blast attribution to different leukocyte lineage.

The blast enumeration and identification are performed separately by morphology and cytochemistry in a bone marrow aspirate film and by surface and intracellular CD determination by flow cytometry after gating by side-scattering properties and the levels of CD45 expression. The results from both methods are then compared and integrated to establish the presence of acute leukemia and to
Volume 2 Issue 3 - 2018

\author{
Sofya Kuznetsova, ${ }^{1,2}$ Olga Fedyanina ${ }^{1,2}$ \\ 'National Research Centre for Pediatric Hematology, Oncology \\ and Immunology, Russia \\ ${ }^{2}$ Centre for Theoretical Problems of Physico-Chemical \\ Pharmacology, Russia
}

Correspondence: Sofya Kuznetsova, National Research

Centre for Pediatric Hematology, Oncology and Immunology, Samory Mashela st. I, Moscow, I I 7997, Russia, Tel +7(9I7)508-

45-50,Email kuznetsova.sonya@gmail.com

Received: April 25, 2018 | Published: May 08, 2018

determine its subtype (ALL BI-BIV, ALL TI-TIV or AML M0-M7). As morphology and flow cytometry use different methods to define the blast population, blasts detected by these two methods are not necessarily the same. ${ }^{5,6}$ This fact can lead to differences in the size of blast population determined by morphology and flow cytometry, as well as pose problems in attributing the leukemic cells to different lineages and determining the leukemia subtype.

To solve this problem and to integrate the morphology and immunophenotyping in the acute leukemia diagnosis we would need a method permitting the morphology study and the surface clusterof-differentiation antigen determination on the same cells. Several approaches have been tried to this end. Imaging flow cytometry is a beautiful technique that has a great potential, however, it is not yet able to enumerate cells with blast morphology. ${ }^{7,8}$ Immunocytochemistry, although permitting to visualize the cells expressing certain surface or intracellular antigens in a film, is also unsuitable for morphology studies. The most suitable for leukemia diagnosis is a technique using a cell-binding anti-CD antibody microarray. Several groups have developed cell-binding anti-CD microarrays capable of "panning" the cells by their surface $\mathrm{CD}$ antigens and determining relative content of the cells positive for certain $\mathrm{CD}$ antigens in analyzed samples..$^{9-11}$ However, making the microarray-bound cells suitable for morphology and/or cytochemistry analysis posed serious technical problems.

We have previously reported the development of an anti-CD antibody microarray on a transparent plastic slide for leukocyte sorting and a method for preparation of the microarray-bound mononuclear cells for high-resolution morphology analysis. ${ }^{12}$ The microarray consisted of monoclonal anti-CD antibody spots $1 \mathrm{~mm}$ in diameter adsorbed on a polyvinylchloride slide blocked with 5\% BSA solution. The mononuclear cell fraction of peripheral blood or bone marrow aspirate is incubated on the microarray; the unbound cells are washed away and the microarray with the antibody-bound cells are dried in a home-made cytocentrifuge. After that the microarray is stained for morphology or cytochemistry using the standard protocols developed for the leukocyte films. ${ }^{12}$ We have shown that the cell binding to the microarray is highly specific and both normal and 
leukemic microarray-bound cells are morphologically identical to the same cells in films.

The close correlation between the leukocyte immunophenotype, morphology and cytochemistry determined by the anti-CD microarray and by standard diagnostic methods suggests a strategy for the microarray application in acute leukemia diagnosis:

i. Using the positive control (cells bound to an antibody to panleukocyte surface marker) determine whether the percentage of cells with blast morphology is above the acute leukemia threshold. The 20\% blast threshold used for bone marrow aspirate films cannot be used if only the mononuclear cell fraction is analyzed and has to be determined separately.

ii. Check all the antibody spots for the presence of blasts. The list of the $\mathrm{CD}$ antigens, the antibodies against which bind the cells with blast morphology constitutes the blast immunophenotype.

iii. Use blast morphology, immunophenotype and cytochemistry (if necessary) to define blast lineage and determine acute leukemia subtype.

To check the viability of the proposed scheme we have studied the mononuclear cell fractions of bone marrow aspirates of 177 children from 0 to 18 years old with suspected acute leukemia, 3 patients with chronic myeloid leukemia, 9 patients with juvenile myelomonocytic leukemia, 4 patients with AML in remission and 20 healthy volunteers using the microarray including the monoclonal antibodies against CD1a, CD2, CD3, CD4, CD5, CD7, CD8a, CD10, CD11b, CD11c, CD13, CD14, CD15, CD16, CD19, CD20, CD22, CD23, CD25, CD33, CD38, CD41, CD45, CD45RA, CD45RO, CD56, CD61a, CD64, CD103, CD117, CD123, CD200, CD235, kappa and lambda immunoglobulin light chains, HLA-DR, IgM and mouse $\operatorname{IgG} 1$ isotype control. Using the training set of 59 patients with known acute leukemia diagnosis, all the patients with chronic leukemias and normal controls we have determined the blast threshold for the acute leukemia diagnosis is $25 \%$; the patients with more than $25 \%$ blasts among the anti-CD45RA-captured cells can be supposed to have acute leukemia with $94 \%$ sensitivity and $97 \%$ specificity. Then, using the same acute leukemia training set we have developed the criteria for classifying leukemia into BI-BIV ALL, TI-TIV ALL and AML M0-M7 using the blast immunophenotype. Finally, using the test set of 74 patients with suspected acute leukemia and applying the developed criteria we have attempted to diagnose the patients blindly in parallel with standard diagnostic procedures.

\section{Conclusion}

Our results show that the presence or absence of acute leukemia and $\mathrm{AL}$ attribution to B-cell, T-cell or myeloid lineage and B-ALL or T-ALL subtype diagnosis using the anti-CD antibody microarray and above described algorithm are made with $100 \%$ accuracy. Among 32 AML patients 2 were diagnosed with AML M4 by microarray while standard diagnostic procedure resulted in AML M5 diagnosis. For all the other AML patients the diagnosis by anti-CD microarray coincided with the final diagnosis. The results of this research project will be reported in detail elsewhere.

Morphology and cytochemistry are more subjective then most diagnostic methods used in acute leukemia diagnosis and are highly dependent on the analyst experience and qualification. Nevertheless they are still indispensable due to their low cost and uniform availability as a first step of acute leukemia diagnosis. Performing morphology and/or cytochemistry on the anti-CD antibody microarray instead of (or in parallel to) the bone marrow aspirate films is a step up on conventional morphology largely increasing the amount and reliability of information on the studied cells while retaining the low cost of the analysis and giving a second chance to these time-tested methods.

\section{Acknowledgements}

The work was partially supported by grant \#18-015-00272 from Russian Foundation for Basic Research.

\section{Conflict of interest}

Author declares no conflict of interest.

\section{References}

1. Arber DA, Orazi A, Hasserjian R, et al. The 2016 revision to the World Health Organization (WHO) classification of myeloid neoplasms and acute leukemia. Blood. 2016;127(20):2391-2405.

2. Bennett JM, Catovsky D, Daniel MT, et al. Proposal for the recognition of minimally differentiated acute myeloid leukaemia (AML-MO). Br J Haematol. 1991;78(3):325-329.

3. Creutzig U, Van Den Heuvel-Eibrink MM, Gibson B, et al. Diagnosis and management of acute myeloid leukemia in children and adolescents: recommendations from an international expert panel. Blood. 2012;120(16):3187-3205.

4. Hoelzer D, Bassan R, Dombret H, et al. Acute lymphoblastic leukaemia in adult patients: ESMO Clinical Practice Guidelines for diagnosis, treatment and follow-up. Ann Oncol. 2016;27(suppl 5):69-82.

5. Harrington AM, Olteanu H, Kroft SH. A dissection of the CD45/side scatter "blast gate". Am J Clin Pathol. 2012;137(5):800-804.

6. Mufti GJ, Bennett JM, Goasguen J, et al. Diagnosis and classification of myelodysplastic syndrome: International Working Group on Morphology of myelodysplastic syndrome (IWGM-MDS) consensus proposals for the definition and enumeration of myeloblasts and ring sideroblasts. Haematologica. 2008;93(11):1712-1717.

7. Wu $\mathrm{CH}$, Wang $\mathrm{TD}$, Hsieh $\mathrm{CH}$, et al. Imaging cytometry of human leukocytes with third harmonic generation microscopy. Scientific reports. 2016;6:37210.

8. Han Y, Gu Y, Zhang AC, et al. Imaging technologies for flow cytometry Lab Chip. 2016;16(24):4639-4647.

9. Barber N, Gez S, Belov L, et al. Profiling CD antigens on leukaemias with an antibody microarray. FEBS letters. 2009;583(11):1785-1791.

10. He Zhu, Monica Macal, Caroline N Jones, et al. A miniature cytometry platform for capture and characterization of T-lymphocytes from human blood. Anal Chim Acta. 2008;608(2):186-196.

11. Ko IK, Kato K, Iwata H. Antibody microarray for correlating cell phenotype with surface marker. Biomaterials. 2005;26(6):687-696.

12. Khvastunova AN, Kuznetsova SA, Al-Radi LS, et al. Anti-CD antibody microarray for human leukocyte morphology examination allows analyzing rare cell populations and suggesting preliminary diagnosis in leukemia. Scientific reports. 2015;5:12573. 